\title{
Pragmatisme et efficacité
}

Le financement du système éducatif norvégien

Pragmatism and effectiveness. Funding of the Norwegian educational system

La financiación del sistema educativo noruego : el pragmatismo al servicio de la eficacia

\section{Bjørn Stensaker}

Traducteur : Jérôme Quintana

\section{OpenEdition}

\section{Journals}

Édition électronique

URL : http://journals.openedition.org/ries/3705

DOI : 10.4000/ries.3705

ISSN : 2261-4265

\section{Éditeur}

Centre international d'études pédagogiques

\section{Édition imprimée}

Date de publication : 15 avril 2014

Pagination : 143-151

ISBN : 978-2-85420-603-6

ISSN : $1254-4590$

Référence électronique

Bjørn Stensaker, «Pragmatisme et efficacité », Revue internationale d'éducation de Sèvres [En ligne], 65 | avril 2014, mis en ligne le 15 avril 2016, consulté le 06 janvier 2020. URL : http://

journals.openedition.org/ries/3705; DOI : 10.4000/ries.3705 


\section{Pragmatisme et efficacité}

Le financement du système éducatif norvégien *

\section{Bjørn Stensaker}

Cet article se propose de décrire et d'analyser le financement du système éducatif norvégien, en s'intéressant plus particulièrement au lien entre le financement et les questions relatives à la qualité, l'efficacité et l'efficience. Le système éducatif norvégien a pour objectif de compter parmi les meilleurs au monde et affiche des ambitions élevées en matière de qualité, d'efficacité et d'efficience (ministère de l'éducation, 2005). Si les ambitions concernant la qualité et l'efficience sont assez caractéristiques des pays d'Europe de l'Ouest, les questions d'efficacité, en revanche, sont fortement liées à la notion d'équité en termes à la fois géographiques et socio-économiques. De ce fait, on peut dire que le système éducatif est plutôt bien réparti à tous les niveaux, permettant aux Norvégiens d'avoir accès aux diverses offres éducatives près de leur domicile (Ministère de l'éducation, 2005).

\section{LE SYSTÈME ÉDUCATIF NORVÉGIEN : CARACTÉRISTIQUES STRUCTURELLES ET HISTORIQUES}

Sur une population de près de cinq millions d'habitants, plus d'un million de Norvégiens fréquentent actuellement une structure d'enseignement ou de formation, de quelque nature que ce soit. En outre, chaque année, environ un million d'adultes suivent des stages de formation. En général, on considère que le système éducatif relève d'une responsabilité publique financée principalement par le secteur public. Néanmoins, le secteur privé est présent à tous les niveaux du système, même si tous les établissements privés sont à but non lucratif. Sur les plus de six mille établissements préscolaires que compte le pays, environ la moitié relève du secteur privé et reçoit également des fonds publics. Dans les établissements préscolaires tant publics que privés, les parents paient pour la scolarité de leurs enfants, même s'il existe un plafond sur les tarifs (identique pour les établissements publics et privés). Dans le primaire et le secondaire, sur plus de trois mille établissements, seulement $5 \%$ environ sont privés et ils n'accueillent que 2 à $3 \%$ des effectifs. Ces deux cycles sont fondés sur le principe de l'équité et de l'éducation pour tous, en s'adaptant à tous les

* Article traduit par Jérôme Quintana. 
types d'élèves, selon le même curriculum national. L'enseignement primaire et secondaire s'étend au total sur dix années.

On accède à l'enseignement secondaire supérieur [de 16 à 18 ans] à l'issue du cycle de l'enseignement secondaire inférieur [de 13 à 15 ans]. L'enseignement secondaire supérieur mène soit aux études supérieures, soit à l'obtention d'une formation ou d'une qualification professionnelle. Dans ce cycle de l'enseignement, les établissements publics dominent en nombre, même si 15 à $20 \%$ des établissements sont privés. Bien que leur nombre soit relativement élevé, les établissements privés de l'enseignement secondaire supérieur n'accueillent que peu d'élèves (environ $6 \%$ de l'effectif total).

L'enseignement supérieur en Norvège a connu des bouleversements internes aux établissements durant la dernière décennie, plusieurs collèges universitaires ayant été transformés en universités à part entière. Aujourd'hui, l'enseignement supérieur en Norvège se compose de huit universités d'État, neuf établissements universitaires spécialisés (dont trois privés), vingt collèges universitaires d'État et environ vingt petits collèges universitaires privés (qui accueillent un peu plus de $10 \%$ du nombre total d'étudiants). À l'exception de la «BI» (l'École norvégienne de management), qui compte plus de dix mille étudiants, la plupart des établissements privés sont de petite taille, avec des effectifs ne dépassant pas mille étudiants chacun. Au total, il y a près de 240000 étudiants inscrits dans le supérieur. La Norvège a adopté la réforme de Bologne en 2004 et mis en place la structure « $3+2+3 »^{1}$, le système européen de transfert et d'accumulation de crédits (ECTS), ainsi que la nouvelle échelle de notation qui en découle.

Le secteur de l'enseignement supérieur professionnel représente une alternative à l'enseignement supérieur traditionnel. On y accède à l'issue des trois années de l'enseignement secondaire supérieur. Dans ce secteur de l'enseignement supérieur, les formations durent normalement de six mois à deux ans. Mis à part les établissements d'enseignement technique et de formation aux métiers de la mer, financés et gérés par les collectivités publiques à l'échelle du comté, le reste des établissements relève principalement du privé. Toutefois, ce secteur de l'enseignement supérieur est assez faible en termes d'effectifs : il accueille un nombre relativement peu élevé d'élèves dans chacun des établissements (Ministère de l'éducation, 2013).

\section{LA GOUVERNANCE DU SYSTÈME ÉDUCATIF}

Les municipalités sont normalement propriétaires des écoles primaires et secondaires, alors que les pouvoirs publics à l'échelle du comté et de la région sont les principaux propriétaires des établissements du secondaire supérieur. Le ministère de l'éducation, de son côté, est le propriétaire officiel de tous les

1. $3+2+3$ : Licence en trois ans, master en deux ans et doctorat en trois ans. (NdT) 
établissements publics de l'enseignement supérieur. Les pouvoirs publics à l'échelle du comté sont légalement contraints de suivre les jeunes âgés de 16 à 21 ans qui ont décroché de l'enseignement secondaire supérieur et sont sans emploi.

En ce qui concerne les établissements privés, le statut de propriété varie. Au niveau de l'enseignement primaire, notamment, la question de savoir quel statut de propriété privée adopter a donné lieu à des débats houleux ces dernières années. Bien que la politique nationale sur cette question ait varié au gré des changements de coalitions politiques, dans l'ensemble les politiques mises en place concernant les établissements privés du primaire sont assez restreintes. Les gouvernements conservateurs ont une approche plus libérale de la question, autorisant une plus grande diversité de statuts pour les établissements privés, y compris pour les établissements à profil confessionnel. Néanmoins, les établissements souhaitant obtenir un agrément doivent avant tout être de type confessionnel ou proposer une approche pédagogique reconnue qui diffère de celle pratiquée dans les établissements publics. Les établissements internationaux certifiés peuvent également recevoir un agrément, ainsi que les établissements privés qui proposent à l'étranger un enseignement norvégien de niveau primaire et secondaire. Toutefois, même les gouvernements conservateurs n'ont pas autorisé l'ouverture d'établissements à but lucratif, même si des propriétaires privés sont libres d'ouvrir des établissements d'enseignement secondaire supérieur. Mais rares sont les établissements privés de ce type.

Dans l'enseignement supérieur, la gouvernance du système a été de tout temps largement influencée par un ministère de l'éducation très présent, bien que davantage de modes de gouvernance s'inspirant de la nouvelle gestion publique aient été mis en place depuis quelques décennies (Bleiklie, 2009). Même si l'on peut distinguer différents types d'établissements d'enseignement supérieur en Norvège, la gouvernance du secteur de l'enseignement supérieur repose encore sur un système plutôt coordonné et intégré, y compris pour les établissements privés. Historiquement, l'enseignement supérieur a toujours été financé par le secteur public et fondé sur la gratuité des frais de scolarité, tant pour les étudiants norvégiens que pour les étrangers. Il existe une loi commune, valable pour tous les établissements publics de l'enseignement supérieur. Par ailleurs, le transfert de crédits ainsi que la reconnaissance des cursus et des formations de tous les types d'établissements ont un caractère obligatoire, et ne posent aucun problème dans la plupart des cas. De ce fait, il est relativement aisé pour un étudiant de circuler entre le public et le privé.

\section{LE FINANCEMENT DU SYSTÈME ÉDUCATIF}

La Norvège dépense généralement quatre à cinq fois plus que la moyenne des pays de l'OCDE en matière d'éducation. Au niveau de l'enseignement primaire et secondaire, la dépense moyenne par élève est d'environ 95000 couronnes 
norvégiennes $^{2}$ (NOK) par an (Direction de l'enseignement et de la formation, 2012). Toutefois, les municipalités étant propriétaires des établissements primaires et secondaires, les dépenses pour ces deux cycles de l'enseignement varient considérablement d'une municipalité à l'autre, de 65000 NOK à 200000 NOK en moyenne par élève et par an. Cette variation est la conséquence de priorités politiques diverses et de caractéristiques propres à chaque municipalité. En moyenne, on dépense davantage pour l'enseignement secondaire supérieur, à savoir environ 135000 NOK par an et par élève (direction de l'enseignement et de la formation, 2012). Au fil du temps, à tous les niveaux de l'enseignement, ce sont les ressources allouées à l'enseignement adapté et individualisé qui ont augmenté. À cet égard, le pourcentage d'heures allouées à l'enseignement destiné aux élèves à besoins éducatifs spécifiques ne cesse d'augmenter.

Dans l'enseignement primaire, secondaire et secondaire supérieur, la Loi sur l'enseignement privé réglemente les subventions que l'État accorde à ce secteur. Les établissements privés reçoivent des subsides à hauteur de $85 \%$ des frais de fonctionnement des établissements publics. Pour se voir attribuer ces aides, les établissements doivent se conformer aux lois et aux réglementations relatives à la Loi sur l'enseignement privé, ainsi qu'aux termes de l'agrément qui leur a été délivré. Lorsque les demandes d'aide sont examinées, il est fréquent de prendre en compte la structure de l'enseignement public, ainsi que les considérations d'ordre pédagogique. Il arrive que certains établissements privés soient également autorisés à démarrer leur activité sans avoir reçu d'aide de l'État, mais ces établissements ont néanmoins l'obligation de se conformer à la réglementation telle qu'elle est stipulée dans la loi. Donc, à l'échelle de l'ensemble du système éducatif, les contributions des familles, des entreprises et des donateurs étrangers sont modestes à ce niveau de l'enseignement.

Dans l'enseignement supérieur, le système de financement a changé au cours de la décennie écoulée, depuis la mise en place de ce que l'on a appelé la «Réforme qualité » de 2004, qui s'est avérée être également une réponse au processus de Bologne en Europe (Rapport $\left.n^{\circ} 27,2000-2001\right)$. Cette réforme a tenté de répondre à un certain nombre de grands problèmes dans le secteur de l'enseignement supérieur (Ministère de l'éducation, 2005) :

- le besoin de parvenir à une plus grande efficience de l'enseignement supérieur et de la recherche : l'enseignement supérieur norvégien a connu un taux élevé d'abandon d'études et de retard dans l'obtention d'un diplôme ;

- le besoin d'améliorer la qualité de l'enseignement dispensé dans le supérieur : notamment dans les universités, le fait que les étudiants aient peu de contact avec leurs enseignants a posé un problème ; également, le fait que les méthodes utilisées soient dépassées, fondées sur les cours magistraux traditionnels, et qu'on mette peu l'accent sur l'apprentissage par les étudiants ;

2. Une couronne norvégienne équivaut à environ o,12 euro ou o,16 dollar US. (NdT) 
- le besoin d'adapter l'enseignement supérieur norvégien au processus de Bologne en cours, et de prendre en compte les obligations de la Norvège dans ce domaine ;

- le besoin de mettre en place un système de gouvernance de l'enseignement supérieur qui permette à ce dernier de répondre aux défis mentionnés ci-dessus.

Dans le cadre de la "Réforme qualité », les structures institutionnelles de gouvernance ont accordé aux établissements la pleine autonomie en matière d'organisation et de gestion pour tous les niveaux hiérarchiques situés en dessous du conseil d'administration et du président. Fondamentalement, cette autonomie sur le plan de l'organisation impliquait qu'il appartenait désormais à l'établissement d'enseignement supérieur de décider de son propre mode d'organisation et de système de gouvernance.

À la suite de cette réforme, un nouveau régime de financement pour l'enseignement supérieur a également été introduit à partir de l'année 2004. On peut dire que la mise en place de ce régime s'inscrit dans la continuité des changements précédemment opérés en matière de financement du supérieur, en mettant davantage l'accent sur le rendement et la performance du système (Frølich, 2007). Le changement le plus important apporté au système a résidé dans le fait qu'une plus grande part du budget dépendait désormais des résultats obtenus et que divers «indicateurs de performance » ont été introduits. Parmi les éléments clés de ce nouveau système : une nouvelle formule de financement des établissements, mettant l'accent sur l'obtention de résultats et le rendement institutionnel, de manière plus forte que dans les précédents systèmes. Le système de financement comporte trois éléments : l'un pour le financement de base, lié aux effectifs (60\% des ressources allouées) ; un autre pour la recherche (15\% des ressources allouées); un autre, enfin, pour l'enseignement (25\% des ressources allouées). Les deux derniers éléments reposent sur les résultats et sont liés à divers indicateurs de performance (par exemple, des publications dans le cadre de la recherche, des points de crédit gagnés par les étudiants, etc.).

Un nouveau régime, cette fois pour les aides financières attribuées aux étudiants, a également été mis en place afin d'encourager les étudiants à obtenir leur diplôme dans les temps. Le système a été conçu de telle sorte que toute aide financière attribuée aux étudiants ne soit accordée qu'à titre de prêt couvrant l'ensemble des frais liés à la scolarité, la poursuite des études jusqu'au diplôme transformant $40 \%$ du prêt en bourse, ce qui constitue un moyen direct d'inciter les étudiants à terminer leurs études. Les étudiants des établissements privés de l'enseignement supérieur paient des frais de scolarité, bien que ces établissements reçoivent des subsides de l'État. Les frais de scolarité varient selon les établissements et le type de cursus suivi. Il n'existe aucun plafonnement en matière de frais de scolarité dans le secteur privé de l'enseignement supérieur. Comme nous l'indiquions plus haut, il n'y a en revanche pas de frais de scolarité dans le secteur public de l'enseignement supérieur. 


\section{FinANCEMENT, GOUVERNANCE ET OBJECTIFS POLITIQUES : QUELQUES RÉFLEXIONS}

Si l'on excepte les changements apportés au système de financement de l'enseignement supérieur, et notamment le poids accru du rendement et de la performance à ce niveau de l'enseignement, on constate que relativement peu de changements ont eu lieu en matière de financement du système éducatif norvégien. Comme nous l'avons montré plus haut, le financement du système éducatif s'inscrit dans une volonté assez prévisible de répartition des moyens fortement influencée par des objectifs politiques d'équité et de disponibilité géographique de l'offre pédagogique sur l'ensemble du territoire.

Dans l'enseignement primaire, secondaire et secondaire supérieur, les niveaux de financement varient d'une municipalité à l'autre, et d'un comté à l'autre, selon des critères divers mais peu liés aux performances de chaque établissement. En effet, les critères de financement se décident plutôt selon le fait que les municipalités de petite taille sont constituées de petits hameaux épars, augmentant ainsi le nombre de petites écoles, ou encore selon la proportion d'élèves à besoins éducatifs spécifiques ou nécessitant une adaptation individualisée de l'apprentissage (Direction de l'enseignement et de la formation, 2012). De ce fait, on peut de manière générale avancer l'idée que la plupart des critères en matière de répartition des ressources, à ces niveaux de l'enseignement, sont axés sur les moyens mis en œuvre (input-oriented) selon les caractéristiques socio-économiques de la région ou les caractéristiques individuelles des élèves. Cette orientation axée sur les moyens mis en œuvre en matière de critères d'obtention de fonds publics vaut également pour les établissements privés, et le système de financement de ces établissements est très prévisible, même si historiquement les changements de majorité politique ont contribué à changer quelque peu la législation les concernant. Ce système de financement est très lié aux objectifs de politique générale visant à garantir l'équité de la société norvégienne, minimisant les questions d'efficience même si certaines enquêtes internationales (telles que l'enquête PISA) ont contribué à attirer davantage l'attention des responsables politiques sur la question des acquis de l'apprentissage. Cependant, à ce jour, rares sont les tentatives de lier le financement aux performances scolaires des établissements.

Dans l'enseignement supérieur, la situation n'est pas la même que dans les cycles inférieurs du système éducatif. L'augmentation des effectifs ainsi que les réformes du secteur public ont permis d'accroître de manière constante la part du financement fondée sur la performance. Comme nous l'avons indiqué plus haut, près de $40 \%$ de l'enseignement supérieur repose aujourd'hui sur la performance, les taux d'obtention du diplôme et le nombre de points de crédit gagnés servant d'indicateurs clés (Frølich, 2006). 
Si la création d'un établissement privé est assujettie à des restrictions, notamment en termes d'objectifs, la réglementation à laquelle les établissements privés sont soumis est plus souple à mesure que l'on avance dans les échelons supérieurs du système éducatif. Ainsi, la création d'établissements privés d'enseignement supérieur repose sur les mêmes règles que pour le public. Les établissements tant publics que privés reçoivent leur accréditation de la même agence nationale d'assurance qualité (NOKUT), et le statut institutionnel obtenu est le même, si certaines conditions sont remplies. C'est pourquoi, dans l'enseignement supérieur, on constate que les questions relatives à la qualité et à l'efficacité sont de moindre importance dans le système de financement, et que les établissements privés peuvent s'établir dans n'importe quelle région et avoir le profil qu'ils souhaitent, tant que les critères d'accréditation sont satisfaits. Cela ne veut pas pour autant dire que les questions liées à la qualité et à l'efficacité n’ont pas leur place dans le débat politique. Durant la décennie écoulée, ces questions ont occupé une place relativement importante dans l'ordre des priorités politiques (Stensaker \& Michelsen, 2012).

Si nous nous intéressons maintenant aux effets induits par le nouveau système de financement dans l'enseignement supérieur, nous constatons que ceux-ci sont relatifs (Frølich et al., 2010). L'enseignement supérieur en Norvège a amélioré ses performances selon un certain nombre d'indicateurs, par exemple en termes de crédits (ECTS) gagnés et de nombre de diplômés. Plus perceptible encore est l'augmentation constante du nombre de publications scientifiques dans les revues spécialisées soumises à un examen par les pairs durant la décennie écoulée, même si le nombre de citations d'articles n'a pas suivi la même hausse (Ministère de l'éducation, 2013). Ces résultats doivent toutefois être également reliés au fait que le financement global a augmenté au cours de la décennie, quoique de façon non linéaire. Par ailleurs, le financement de la recherche a augmenté plus vite que celui consacré à l'enseignement (Ministère de l'éducation, 2013).

Le système de financement introduit dans le cadre de la Réforme qualité constitue peut-être l'élément qui influe le plus sur le comportement des établissements. Dans une étude sur l'affectation des ressources dans les établissements norvégiens, Frølich (2007, p. 26) a découvert que si des variations interviennent dans la façon dont les établissements réaffectent les ressources en interne, ces variations renforcent la plupart du temps l'influence et le pouvoir des établissements. Si certains établissements reproduisent le système national d'incitation pour en faire bénéficier directement les facultés et les départements qui produisent des résultats, d'autres ont mis en place des modèles qui relèvent davantage d'ambitions stratégiques. Selon les tendances observées, davantage de ressources fondées sur des mesures incitatives et liées à la recherche sont directement affectées en retour aux unités de recherche individuelles, alors que celles liées par exemple aux points de crédit gagnés sont redistribuées à l'échelle de 
l'établissement (Frølich, 2007). Cela veut dire qu'une faculté et un département, pris individuellement, obtiennent de meilleurs retours sur performance dans le domaine de la recherche que dans celui de l'enseignement. Ainsi, recherche et enseignement peuvent également être découplés pour assumer des rôles distincts en termes de gestion, ce qui crée de nouvelles tensions quant à la façon dont l'autorité est répartie dans les établissements.

De manière générale, au cours des dernières décennies, la Norvège a été fortement influencée par toute la rhétorique autour de la nouvelle gestion publique (New Public Management), à tous les niveaux du système éducatif (Bleiklie, 2009). Toutefois, jusqu'à présent, cela n'a guère entraîné la privatisation des services d'éducation. Les établissements d'enseignement privé sont autorisés à tous les niveaux du système éducatif, mais sont soumis à une réglementation très stricte, notamment dans les cycles inférieurs du système. De ce fait, l'enseignement privé ne joue pas un rôle essentiel dans le système éducatif norvégien, excepté pour le préscolaire. Cette rhétorique autour de la nouvelle gestion publique s'est concrétisée non pas par un processus de privatisation, mais en mettant davantage l'accent sur le financement conditionné par la performance dans l'enseignement supérieur (c'est là que le nombre d'étudiants a le plus augmenté). Elle s'est également concrétisée sous la forme d'évaluations et en insistant davantage sur l'obligation de rendre des comptes, à tous les niveaux du système (Stensaker et Harvey, 2013).

En ce qui concerne une autre dimension de la nouvelle gestion publique, à savoir la forte croyance politique dans la décentralisation comme moyen de créer un secteur public plus efficace et efficient, on peut toutefois faire valoir que, si la rhétorique met l'accent sur l'autonomie locale des établissements, une grande partie des moyens et des pouvoirs restent entre les mains des autorités nationales. Il est intéressant de souligner que, traditionnellement, la Norvège a décentralisé une grande partie des pouvoirs, et que la tendance actuelle à tous les niveaux du système est de promouvoir les établissements de grande taille dans le primaire, le secondaire et le secondaire supérieur (Direction de l'enseignement et de la formation, 2012) et, de plus en plus, de favoriser les fusions dans l'enseignement supérieur (Stensaker et Michelsen, 2012). Nous constatons donc une tendance double : d'une part, une rhétorique abondante autour de la décentralisation et du leadership à tous les niveaux, avec la fermeture par les municipalités des établissements de petite taille, et d'autre part, la création d'établissements de plus grande taille à tous les niveaux. Dans ce paysage complexe, on observe également que l'autorité centrale se dissout à mesure que se créent de nouvelles agences d'État à divers niveaux du système, s'intéressant aux questions liées à la qualité et à l'efficacité. 
Le financement du système éducatif norvégien est traditionnellement lié, d'une part à des indicateurs de moyens mis en œuvre, et d'autre part à la nécessité de satisfaire aux principaux objectifs de la nation. C'est la raison pour laquelle, dans l'enseignement préscolaire, primaire, secondaire et secondaire supérieur, les questions d'efficacité liée à l'équité et de répartition socioéconomique des services d'éducation ont été au centre des préoccupations. Dans l'enseignement supérieur, des changements ont eu lieu, associant davantage financement et performance, mais sans se soucier des questions liées à la qualité et à l'efficacité, questions que le système de financement ne prend pas en compte à ce niveau de l'enseignement.

\section{BIBLIOGRAPHIE}

BLEIKLIE I. (2009) : Norway: From tortoise to eager beaver? In Paradeise, C., Reale, E., Bleiklie, I. \& Ferlie, E. (eds.) University governance. Western European comparative perspectives. (pp. 127-152). Dordrecht : Springer.

FRØLICH N. (2007): Iverksetting av insentivbasert finansiering. (Implementing incentive-based funding). Oslo : NIFU STEP.

FRØLICH N. (2006) : Offentlig politikk for høyere utdanning : endringer i finansiering - endringer i faglige prioriteringer (Public policy for higher education. Changes in funding - changes in academic priorities) ? In Michelsen, S. \& Aamodt, P.O. (eds.) Kvalitetsreformen møter virkeligheten (The Quality Reform Implemented) 82-94. Oslo : The Norwegian Research Council.

FRØLICH N., SCHMIDT E.K. \& ROSA M.J. (2010) : Funding systems for higher education and their impacts on institutional strategies and academia. A comparative perspective. International Journal of Educational Management, 24, 7-21.

Ministry of Education and Research (2005) : OECD thematic review of tertiary education. Country background report for Norway. Oslo : Ministry of Education.

Ministry of Education (2013) : Tilstandsrapport for høyere utdanning (Status report for higher education) Oslo : Ministry of Education.

Norwegian Directorate for Education and Training (2012): The Education Mirror 2012. Analysis of primary and secondary education and training in Norway. Oslo: Directorate for Education and Training.

STENSAKER B. \& HARVEY L. (2013) : The accountability dimension in quality assurance : An international comparison. Higher Education Review, 45, 26-40.

STENSAKER B. \& MICHELSEN S. (2012) : Governmental Steering, Reform and the Institutionalization of Student Interest in Higher Education in Norway. European Journal of Higher Education, 2, 20-31.

St. meld. $\mathrm{n}^{\circ} 27$ (2000-2001) : (Rapport No27, 2000-2001) Gjør din plikt - Krev din rett. Kvalitetsreformen i høyere utdanning (Do your duty - demand your rights. The Quality reform in higher education). Oslo : Ministry of Education. 
